\title{
The Egyptian health map: a guide for evidence-based decision- making
}

Ghada Radwan ${ }^{1,2}$ and Adel Adawy ${ }^{3,4}$

${ }^{1}$ Faculty of Medicine, Cairo University, Cairo, Egypt. ${ }^{2}$ Technical Office, Ministry of Health and Population, Cairo, Egypt. ${ }^{3}$ Ministry of Health and Population, Cairo, Egypt. ‘Banha University, Cairo, Egypt. (Correspondence to: Ghada Radwan: ghadanasr@gmail.com).

\begin{abstract}
Background: The Egyptian health system is characterized by the multiple entities in charge of service regulation, financing and provision. Moreover, health outcomes in Egypt mirror epidemiologic transition; increasing prevalence of noncommunicable diseases (NCDs) and persistent high levels of selected communicable diseases (CDs) place a huge financial burden on the health system's limited resources. This together with financial inefficiencies aggravate inequality in health services coverage and health outcomes.

Aims: We aimed to map and link health services with key health outcomes to support efficient utilization of scarce resources and achieving Universal Health Coverage (UHC).

Methods: Data on health services and health outcomes were obtained from the Ministry of Health and Population and key national surveys for the year 2014. Data on health services' availability and coverage were compared in different geographical regions. Correlation analysis was used to test the relation between selected service availability indicators and mortality rates from key NCDs.

Results: There was a high coverage of key maternal and child health services. However, there was in general low achievement of national targets for service availability as well as wide geographical disparities. Cardiovascular diseases were the leading cause of death and there was a marked reduction in the incidence of major CDs in Egypt, particularly vaccine preventable diseases.

Conclusion: : Wide geographical disparities and increased burden of NCDs and their risk factors are identified as key challenges facing the healthcare system in Egypt. Priority setting in the light of these findings is crucial to support national efforts to attain health equity and UHC.

Keywords: Health systems, morbidity, mortality, fertility, Egypt

Citation: Radwan G; Adawy A. The Egyptian health map: a guide for evidence-based decision-making. East Mediterr Health J. 2019;25(5):350-361 https:// doi.org/10.26719/emhj.18.048

Received: 02/10/17; accepted: 20/05/18

Copyright ( ) World Health Organization (WHO) 2019. Some rights reserved. This work is available under the CC BY-NC-SA 3.0 IGO license (https:// creativecommons.org/licenses/by-nc-sa/3.o/igo).
\end{abstract}

\section{Introduction}

Egypt has a highly pluralistic healthcare system with many different public and private providers and financing bodies (1). The governmental health sector comprises primarily the Ministry of Health and Population (MoHP) and university hospitals. MoHP manages the primary healthcare centres and units, general hospitals, district hospitals, and integrated and specialized hospitals (1). The level of government health expenditure is low, representing only $2 \%$ of Gross Domestic Product (GDP). However, the 2014 constitution affirmed the universal right to healthcare and that the state shall allocate a percentage of GDP on health not less than 3\%, a value that represents almost twice the current governmental expenditure on the health sector (2).

Increased budgetary allocations for health are dependent on the health sector's ability to demonstrate good use of its resources including equitable distribution of available resources (2). Information on health system performance and on key factors that explain performance variation can strengthen the scientific foundations of health policy, provide important insights into effective policies and consequently pave the road for Universal Health Coverage UHC (3).

The aim of the current study was to map health service availability, coverage and key health outcomes and examine the association between health services availability indicators and mortality rates including Maternal Mortality Ratio (MMR). The ultimate goal was to identify major gaps in health services and outcomes for better allocation of scarce resources, promotion of health equity and attaining UHC.

\section{Methods}

The main sources of data were the Health Information Center at MoHP, the Egyptian Demographic Health Survey (EDHS) 2014, and National Cancer Registry Program of Egypt (NCRPE) $(4,5)$. Indicators were defined based on the World Health Organization (WHO) World Health Statistics 2015: Indicator compendium (6). These included general service availability $(6,7)$, health services coverage (6-8), health systems statistics (8), and mortality, morbidity and fertility indicators (6). 
General service availability and coverage indicators were calculated from data collected from all sectors except for general hospitals (only available at MoHP), intensive care units (ICUs), neonatal intensive care unit (NICUs) and ambulances (disaggregated data available only from MoHP). They were compared relative to national benchmarks developed by MoHP (internal documents) and included:

\section{- One General hospital / 500000 population}

- One intensive care unit / 15000 population

- One neonatal intensive care unit/400 live births

- One ambulance / 25000 population

MoHP Institutional Review Board approval was obtained prior to data collection and analysis. Indicators were disaggregated by geographical area in Egypt; either a governorate or region depending on the availability of data (9). Skewed data were identified using KolmogorovSmirnov test. ANOVA test or Kruskal-Wallis test was conducted to examine differences in service availability indicators as well as mortality indicators across different geographical regions. Correlation analysis was used to test correlation between selected service availability indicators and mortality rates from key NCDs and MMR. $\mathrm{P}$ - value $<0.05$ was considered statistically significant. SPSS version 21 was used for data analysis.

\section{Results}

\section{General service availability}

Overall, there was 0.23 health facility per 10000 population in 2014 compared to 0.37 in the year 2001, representing a 38\% reduction over the past 14 years (data are not shown). The number of beds in the public sector represented nearly three quarters of the total number of beds in 2014 and there were 15 beds for every 10000 Egyptians in 2014. For primary healthcare (PHC) facilities, there were 0.59 PHC facility/ 10000 of the population.

There were approximately $35 \%, 65 \%, 27 \%$ and $38 \%$ achievement of national targets regarding general hospitals, ambulances, ICUs and NICUs respectively. Wide geographical disparities were clearly evident (Table 1) and demonstrated by the statistical significant

\begin{tabular}{|c|c|c|c|c|c|c|c|}
\hline Governorates & $\begin{array}{c}\text { Percent } \\
\text { achievement } \\
\text { of the target } \\
\text { general } \\
\text { hospital } \neq\end{array}$ & $\begin{array}{c}\text { Percent } \\
\text { achievement } \\
\text { of the target } \\
\text { ambulances } \$\end{array}$ & $\begin{array}{c}\text { Percent } \\
\text { achievement } \\
\text { of target } \\
\text { ICUs }\end{array}$ & $\begin{array}{c}\text { Percent } \\
\text { achievement } \\
\text { of target } \\
\text { NICUs } \$\end{array}$ & $\begin{array}{c}\text { Health } \\
\text { facility/10000 } \\
\text { population }\end{array}$ & $\begin{array}{l}\text { Beds/10 } 000 \\
\text { population }\end{array}$ & $\begin{array}{c}\text { Primary } \\
\text { health care } \\
\text { facilities/1000o } \\
\text { population } \neq\end{array}$ \\
\hline Cairo & 70.1 & 70.3 & 16.7 & 19.8 & 0.5 & 34.4 & 0.2 \\
\hline Alexandria & 31.2 & 38.4 & 8.7 & 12.2 & 0.3 & 27.1 & 0.3 \\
\hline Port-Said & 75.0 & 161.3 & 184.5 & 141.6 & 0.4 & 22.0 & 0.5 \\
\hline Suez & 80.3 & 244.8 & 40.9 & 42.3 & 0.5 & 17.8 & 0.5 \\
\hline Ismailia & 42.4 & 161.2 & 35.6 & 58.4 & 0.5 & 33.4 & 0.5 \\
\hline Damietta & 75.1 & 93.9 & 71.0 & 126.1 & 0.4 & 14.5 & 0.7 \\
\hline Dakahlia & 25.2 & 32.8 & 40.3 & 70.9 & 0.1 & 4.6 & 0.8 \\
\hline Sharkia & 23.1 & 50.9 & 31.0 & 45.6 & 0.3 & 14.2 & 0.7 \\
\hline Kalyoubia & 9.8 & 36.7 & 11.8 & 13.4 & 0.2 & 12.8 & 0.4 \\
\hline Kafr-ElSheikh & 31.5 & 52.0 & 23.6 & 49.3 & 0.3 & 24.0 & 0.8 \\
\hline Gharbia & 42.1 & 30.5 & 29.4 & 22.1 & 0.1 & 5.5 & 0.6 \\
\hline Menoufia & 25.4 & 35.5 & $29 \cdot 3$ & 26.7 & 0.3 & 17.2 & 0.6 \\
\hline Behera & 8.6 & 31.4 & 19.4 & 37.8 & 0.1 & 8.7 & 0.7 \\
\hline Giza & 33.0 & 49.1 & 17.6 & 17.4 & 0.3 & 11.9 & 0.3 \\
\hline Beni-Suef & $17 \cdot 5$ & 57.8 & 23.6 & 52.5 & 0.1 & 8.4 & 0.7 \\
\hline Fayoum & 15.8 & 48.9 & 22.7 & 32.3 & 0.1 & 6.5 & 0.5 \\
\hline Menia & 19.4 & 34.4 & 18.3 & 30.6 & 0.1 & 8.0 & 0.7 \\
\hline Asyout & $35 \cdot 3$ & 50.6 & 25.4 & 38.4 & 0.2 & 17.1 & 0.6 \\
\hline Suhag & 32.6 & 44.5 & 20.2 & 35.8 & 0.1 & 8.4 & 0.7 \\
\hline Qena & 32.8 & 58.3 & 26.6 & 43.0 & 0.1 & $5 \cdot 3$ & 0.8 \\
\hline Aswan & 34.9 & 139.7 & 24.1 & 55.0 & 0.2 & 14.1 & 1.5 \\
\hline
\end{tabular}




\begin{tabular}{|c|c|c|c|c|c|c|c|}
\hline Governorates & $\begin{array}{c}\text { Percent } \\
\text { achievement } \\
\text { of the target } \\
\text { general } \\
\text { hospital } \$\end{array}$ & $\begin{array}{c}\text { Percent } \\
\text { achievement } \\
\text { of the target } \\
\text { ambulances } \ddagger\end{array}$ & $\begin{array}{c}\text { Percent } \\
\text { achievement } \\
\text { of target } \\
\text { ICUsł }\end{array}$ & $\begin{array}{c}\text { Percent } \\
\text { achievement } \\
\text { of target } \\
\text { NICUs } \ddagger\end{array}$ & $\begin{array}{c}\text { Health } \\
\text { facility/10000 } \\
\text { population }\end{array}$ & $\begin{array}{l}\text { Beds/10 ooo } \\
\text { population }\end{array}$ & $\begin{array}{c}\text { Primary } \\
\text { health care } \\
\text { facilities } / 10000 \\
\text { population } \neq\end{array}$ \\
\hline Matrouh & 111.7 & 547.1 & 130.6 & 24.5 & 0.3 & 9.7 & 1.2 \\
\hline El Wadi ElGidid & 221.8 & 787.4 & 113.1 & 128.7 & 1.0 & 35.9 & 2.3 \\
\hline Red Sea & 144.6 & 629.0 & 99.8 & 110.3 & 0.4 & $17.9 \mathrm{w}$ & 0.8 \\
\hline North Sinai & 115.0 & 460.0 & 113.9 & 110.0 & 0.3 & 11.3 & 1.9 \\
\hline South Sinai & 298.6 & 1403.6 & 250.9 & $347 \cdot 3$ & 0.8 & 66.8 & 1.4 \\
\hline Luxor & 43.6 & 152.6 & 57.5 & 89.2 & 0.2 & 9.2 & 1.0 \\
\hline Total & 34.7 & 64.5 & 27.1 & 37.8 & 0.23 & 14.9 & 0.59 \\
\hline
\end{tabular}

\begin{tabular}{|c|c|c|c|c|c|c|}
\hline $\begin{array}{l}\text { Region(\# of Governorates/ } \\
\text { region) }\end{array}$ & $\begin{array}{c}\text { Percent } \\
\text { achievement } \\
\text { of the target } \\
\text { general } \\
\text { hospitals\#* }\end{array}$ & $\begin{array}{c}\text { Percent } \\
\text { achievement } \\
\text { of the target } \\
\text { ambulances\#* }\end{array}$ & $\begin{array}{c}\text { Percent } \\
\text { achievement } \\
\text { of the target } \\
\text { ICUs\#* }\end{array}$ & $\begin{array}{c}\text { Percent } \\
\text { achievement } \\
\text { of the target } \\
\text { NICUs\# }\end{array}$ & $\begin{array}{c}\text { Health } \\
\text { facility/10000 } \\
\text { population\#* }\end{array}$ & $\begin{array}{c}\text { Primary } \\
\text { health care } \\
\text { facilities } / 10000 \\
\text { Population +* }\end{array}$ \\
\hline \multicolumn{7}{|l|}{ Urban (6) } \\
\hline Mean & 55.3 & 120.9 & 50.7 & 48.6 & 0.42 & 0.38 \\
\hline SD & 22.3 & 81.4 & 66.7 & 48.8 & 0.09 & 0.13 \\
\hline \multicolumn{7}{|l|}{ Upper Egypt(7) } \\
\hline Mean & 26.9 & 62.0 & 23.0 & 41.1 & 0.13 & 0.78 \\
\hline SD & 8.9 & 35.2 & 2.9 & 9.6 & 0.04 & 0.33 \\
\hline \multicolumn{7}{|l|}{ Lower Egypt(8) } \\
\hline Mean & 30.1 & 45.5 & 32.0 & 49.0 & 0.23 & 0.66 \\
\hline $\mathrm{SD}$ & 21.2 & 21.3 & 17.9 & 35.9 & 0.12 & 0.13 \\
\hline \multicolumn{7}{|l|}{ Frontiers(6) } \\
\hline Mean & 155.9 & 663.3 & 127.6 & 135.0 & 0.50 & 1.43 \\
\hline SD & 90.7 & 419.6 & 65.2 & 110.1 & 0.32 & 0.56 \\
\hline Total(27) & 62.8 & 203.8 & 55.1 & 66.0 & 0.30 & 0.80 \\
\hline Mean & 67.1 & 314.6 & 58.5 & 67.7 & 0.22 & 0.48 \\
\hline
\end{tabular}

$\ddagger$ Data for MOHP facilities

* $=$ P-value $<0.05$

\# Kruskal-Wallis Test

+ ANOVA Test

Source: MOHP and CAPMAS Health Statistics, 2014

differences in the percent achievement of the national benchmarks in the different geographical regions.

\section{Health service coverage}

There was a high coverage of maternal and child health $(\mathrm{MCH})$ care services and immunization services (4). However, only $57 \%$ of women during child bearing period use modern methods of contraception (Table 2).

\section{Health system statistics}

The labour force in the public sector was estimated at 72 900 physicians (8.4 physicians / 10000 population), and 126200 nurses (14.5 nurses / 10000 population) (9). Data on health expenditure highlighted a stagnation of relevant indicators. For example, total health expenditure (THE) as a percentage of GDP and out of pocket (OOP) stood at $6 \%$ and $56 \%$ respectively $(10,11)$.

\section{Mortality}

Nearly half of deaths were attributed to cardiovascular diseases including ischemic heart diseases, hypertension, and cerebrovascular diseases. Geographical distribution of NCDs deaths / 100000 population revealed significant geographical differences. There were negative correlations between all service availability indicators 
and deaths from NCDs and MMR. For example, deaths attributed to ischemic heart diseases were significantly negatively correlated with percent achievement of the target number of general hospitals, ambulances and ICUs (Table 3, Table 4).

\section{Morbidity}

Data for 2014 revealed that the majority of CDs under MoHP surveillance had an incidence rate below one case/ 100000 population (16 diseases out of 30). Only two CDs had incidence rates above 10 cases/100 000 population (urinary and intestinal bilharziasis, at 54 cases/ 100000 and 26 cases/ 100000 respectively; data are not shown).
The overall prevalence rates of HBV and HCV were $1 \%$ and $4.4 \%$ respectively in the population aged $1-59$ years. $\mathrm{HCV}$ showed the highest prevalence in Lower Egypt region reaching nearly $6 \%$ (Table 5 ).

The crude cancer rate for Egypt was 113/100 000 population with the highest cancer incidence rate reported in Lower Egypt 134/100 ooo population (Table 5). Nearly half of the adult men were current smokers (46.4\%) compared to a very low percentage among women (0.2\%). However, nearly half of women $(50 \cdot 3 \%)$ and one third of men $(26 \cdot 4 \%)$ suffered from obesity. One in five Egyptians suffered from elevated blood pressure (Table 5).

\begin{tabular}{|c|c|c|c|c|c|c|}
\hline Governorate & $\begin{array}{c}\text { Regular } \\
\text { antenatal care } \\
\text { (4 visits or } \\
\text { more) (\%) }\end{array}$ & $\begin{array}{l}\text { Births delivered } \\
\text { by skilled } \\
\text { provider (\%) }\end{array}$ & $\begin{array}{c}\text { Use modern } \\
\text { Contraceptive } \\
\text { method (CPR) } \\
(\%)\end{array}$ & $\begin{array}{l}\text { Total fertility } \\
\text { rate (TFR) } \\
\text { (child/ woman) }\end{array}$ & $\begin{array}{c}\text { Age at first } \\
\text { marriage } \\
\text { (Mean) }\end{array}$ & $\begin{array}{l}\text { Beds/10000 } \\
\text { population }\end{array}$ \\
\hline Cairo & 89.9 & 97.8 & 61.7 & 2.6 & 22.8 & 88.0 \\
\hline Alexandria & 92.7 & 96.0 & 58.9 & 2.2 & 23.4 & 97.3 \\
\hline Port-Said & 96.8 & 99.6 & 56.7 & 3.0 & 23.2 & 95.9 \\
\hline Suez & 89.0 & 99.3 & 60.1 & 3.2 & 22.5 & 88.9 \\
\hline Damietta & 94.6 & 98.9 & 64.2 & 3.0 & 21.0 & 89.9 \\
\hline Dakahlia & 93.1 & 98.9 & 63.2 & 3.1 & 20.4 & 92.5 \\
\hline Sharkia & 83.5 & 92.2 & 57.4 & 3.6 & 20.5 & 82.3 \\
\hline Kalyoubia & 80.1 & 94.2 & 61.7 & 3.8 & 20.9 & 87.2 \\
\hline Kafr-ElSheikh & 91.0 & 98.5 & 62.1 & 3.4 & 21.0 & 100.0 \\
\hline Gharbia & 83.3 & 95.7 & 62.0 & 3.1 & 21.4 & 90.6 \\
\hline Menoufia & 89.0 & 95.1 & 65.6 & 3.5 & 21.2 & 93.0 \\
\hline Behera & 88.0 & 92.8 & 65.5 & 3.5 & 20.6 & 90.8 \\
\hline Ismailia & 85.3 & 95.7 & 58.8 & 3.7 & 22.2 & 93.8 \\
\hline Giza & 79.6 & 93.1 & 62.4 & 3.3 & 20.3 & 86.1 \\
\hline Beni-Suef & 74.8 & 80.7 & $55 \cdot 3$ & 3.9 & 19.5 & 91.8 \\
\hline Fayoum & 75.6 & 84.5 & 55.5 & 4.6 & 19.3 & 96.1 \\
\hline Menia & 70.2 & 73.5 & 48.5 & 3.9 & 19.0 & 88.5 \\
\hline Asyout & 76.7 & 82.4 & 39.5 & 4.2 & 20.2 & 91.9 \\
\hline Suhag & 70.3 & 87.3 & 29.4 & 4.3 & 20.4 & 75.2 \\
\hline Qena & 73.2 & 90.6 & 37.1 & 3.7 & 19.9 & 78.3 \\
\hline Aswan & 85.7 & 98.1 & 47.8 & 3.6 & 20.8 & 92.8 \\
\hline Luxor & 83.7 & 97.9 & 47.2 & 3.4 & 20.4 & 85.2 \\
\hline Red Sea & 86.0 & 94.0 & $55 \cdot 3$ & 3.4 & 22.1 & 91.3 \\
\hline ElWadi ElGidid & 90.2 & 98.3 & 64.5 & 3.7 & 20.9 & 84.2 \\
\hline Matrouh & 59.9 & 78.0 & 40.0 & 4.8 & 19.7 & 93.7 \\
\hline Total & 82.8 & 91.5 & 56.9 & 3.5 & 20.8 & 88.7 \\
\hline
\end{tabular}




\begin{tabular}{|c|c|c|c|c|c|c|}
\hline $\begin{array}{l}\text { Region(\# of } \\
\text { Governorates/region) }\end{array}$ & $\begin{array}{c}\text { Regular } \\
\text { antenatal care } \\
\text { (4 visits or } \\
\text { more) }(\%)+^{*}\end{array}$ & $\begin{array}{c}\text { Births } \\
\text { delivered } \\
\text { by skilled } \\
\text { provider (\%)+* }\end{array}$ & $\begin{array}{l}\text { Use modern } \\
\text { contraceptive } \\
\text { method (CPR)\#* }\end{array}$ & $\begin{array}{c}\text { Total } \\
\text { fertility rate } \\
\text { (TFR) } \#^{*}\end{array}$ & $\begin{array}{l}\text { Age at first } \\
\text { marriage } \\
\text { (Mean)+* }\end{array}$ & $\begin{array}{l}\text { All vaccines and } \\
\text { HBV (\%)+ }\end{array}$ \\
\hline \multicolumn{7}{|l|}{ Urban (6) } \\
\hline Mean & 88.9 & 96.9 & 59.8 & 3.0 & 22.4 & 91.7 \\
\hline SD & 6.0 & 2.5 & 2.1 & 0.5 & 1.1 & 4.6 \\
\hline \multicolumn{7}{|l|}{ Upper Egypt(7) } \\
\hline Mean & 75.2 & 85.3 & 44.7 & 4.0 & 19.9 & 87.8 \\
\hline SD & 5.3 & 7.8 & 9.8 & 0.4 & 0.6 & 7.9 \\
\hline \multicolumn{7}{|l|}{ Lower Egypt(8) } \\
\hline Mean & 87.8 & 95.8 & 62.7 & 3.4 & 20.9 & 90.8 \\
\hline SD & 5.1 & 2.7 & 2.6 & 0.3 & 0.3 & 5.0 \\
\hline \multicolumn{7}{|l|}{ Frontiers(4) } \\
\hline Mean & 80.0 & 92.1 & 51.8 & 3.8 & 20.8 & 88.6 \\
\hline SD & 13.6 & 9.6 & 10.6 & 0.7 & 1.0 & 4.6 \\
\hline $\begin{array}{l}\text { Total }(25)^{* *} \\
\text { Mean }\end{array}$ & 83.3 & 92.5 & 55.2 & 3.5 & 20.9 & 89.8 \\
\hline SD & 9.0 & 7.3 & 9.9 & 0.6 & 1.2 & 5.7 \\
\hline
\end{tabular}

\section{+ ANOVA Test}

\# Kruskal-Wallis Test

${ }^{*}=$ P-value $<0.05$

**Two Governorates (North and South Sinai) were not included in EDHS, 2014

$\ddagger$ Source: EDHS 2014

\begin{tabular}{|c|c|c|c|c|c|c|c|}
\hline Governorates & Cancer & $\begin{array}{l}\text { Chronic } \\
\text { respiratory } \\
\text { diseases }\end{array}$ & $\begin{array}{c}\text { Ischemic } \\
\text { heart } \\
\text { diseases }\end{array}$ & $\begin{array}{c}\text { Hypertensive } \\
\text { diseases }\end{array}$ & $\begin{array}{l}\text { Cerebro- } \\
\text { vascular } \\
\text { diseases }\end{array}$ & $\begin{array}{c}\text { Liver } \\
\text { cirrhosis }\end{array}$ & Road accidents \\
\hline Cairo & 83.0 & 42.4 & 44.0 & 70.2 & 51.0 & 22.0 & 17.0 \\
\hline Alexandria & 107.0 & 26.5 & 51.0 & 114.4 & 55.0 & 17.0 & 18.0 \\
\hline Port-Said & 77.0 & 48.3 & 19.0 & 9.0 & 70.0 & 8.0 & 5.0 \\
\hline Suez & 42.0 & 4.7 & 12.0 & 149.0 & 38.0 & 24.0 & 48.0 \\
\hline Ismailia & 41.0 & 17.7 & 15.0 & 31.1 & 17.0 & 16.0 & 37.0 \\
\hline Damietta & 75.0 & 2.6 & 6.0 & 145.6 & 51.0 & 69.0 & 12.0 \\
\hline Dakahlia & 37.0 & 10.5 & 20.0 & 40.7 & 46.0 & 49.0 & 12.0 \\
\hline Sharkia & 16.0 & 5.0 & 10.0 & 35.5 & 51.0 & 33.0 & 10.0 \\
\hline Kalyoubia & 22.0 & 14.5 & 39.0 & 19.7 & 39.0 & 43.0 & 5.0 \\
\hline Kafr-ElSheikh & 25.0 & 36.3 & 16.0 & 39.0 & 28.0 & 65.0 & 7.0 \\
\hline Gharbia & 24.0 & 70.1 & 14.0 & 40.3 & 46.0 & 41.0 & 4.0 \\
\hline Menoufia & 18.0 & 25.7 & 27.0 & 30.9 & 46.0 & 23.0 & 9.0 \\
\hline Behera & 33.0 & 11.9 & 17.0 & 14.5 & 37.0 & 25.0 & 15.0 \\
\hline Giza & 41.0 & 26.1 & 17.0 & 19.4 & 45.0 & 20.0 & 14.0 \\
\hline Beni-Suef & 19.0 & 17.9 & 39.0 & 5.9 & 85.0 & 48.0 & 11.0 \\
\hline Fayoum & 19.0 & 30.2 & 10.0 & 20.8 & 31.0 & 15.0 & 4.0 \\
\hline Menia & 26.0 & 24.2 & 61.0 & 18.7 & 53.0 & 41.0 & 12.0 \\
\hline Asyout & 30.0 & 40.9 & 14.0 & 12.0 & 47.0 & 31.0 & 17.0 \\
\hline Suhag & 21.0 & 25.5 & 29.0 & 14.6 & 48.0 & 29.0 & 10.0 \\
\hline Qena & 13.0 & 11.7 & 10.0 & 22.1 & 52.0 & 18.0 & 6.0 \\
\hline Aswan & 19.0 & 33.3 & 8.0 & 22.1 & 33.0 & 15.0 & 19.0 \\
\hline
\end{tabular}


Table 3. Deaths / 100000 population from key noncommunicable diseases by governorates, 2014 (concluded)

\begin{tabular}{lccccccc} 
Governorates & Cancer & $\begin{array}{c}\text { Chronic } \\
\text { respiratory } \\
\text { diseases }\end{array}$ & $\begin{array}{c}\text { Ischemic } \\
\text { heart } \\
\text { diseases }\end{array}$ & $\begin{array}{c}\text { Hypertensive } \\
\text { diseases }\end{array}$ & $\begin{array}{c}\text { Cerebro- } \\
\text { vascular } \\
\text { diseases }\end{array}$ & $\begin{array}{c}\text { Liver } \\
\text { cirrhosis }\end{array}$ & Road accidents \\
Matrouh & 8.0 & 14.1 & 2.0 & 2.9 & 14.0 & 0.0 & 1.0 \\
El Wadi ElGidid & 19.0 & 21.3 & 4.0 & 7.1 & 39.0 & 6.0 & 20.0 \\
Red Sea & 11.0 & 59.9 & 12.0 & 4.6 & 48.0 & 4.0 & 44.0 \\
North Sinai & 11.0 & 18.4 & 18.0 & 4.4 & 39.0 & 5.0 & 12.0 \\
South Sinai & 2.0 & 118.9 & 10.0 & 3.0 & 14.0 & 2.0 & 90.0 \\
Luxor & 17.0 & 54.0 & 8.0 & 26.9 & 73.0 & 15.0 & 10.0 \\
Total & $\mathbf{3 7 . 0}$ & $\mathbf{2 6 . 5}$ & $\mathbf{2 6 . 0}$ & $\mathbf{3 6 . 5}$ & $\mathbf{4 7 . 0}$ & $\mathbf{3 0 . 0}$ & $\mathbf{1 2 . 0}$ \\
\hline
\end{tabular}

\begin{tabular}{|c|c|c|c|c|c|c|c|}
\hline $\begin{array}{l}\text { Region(\# of } \\
\text { Governorates/region) }\end{array}$ & $\begin{array}{l}\text { Cancer } \\
\text { deaths \#* }\end{array}$ & $\begin{array}{l}\text { Chronic } \\
\text { respiratory } \\
\text { diseases \# }\end{array}$ & $\begin{array}{c}\text { Ischemic } \\
\text { heart } \\
\text { diseases \# }\end{array}$ & $\begin{array}{l}\text { Hypertensive } \\
\text { diseases \#* }\end{array}$ & $\begin{array}{l}\text { Cerebro- } \\
\text { vascular } \\
\text { diseases+ }\end{array}$ & $\begin{array}{l}\text { Liver } \\
\text { cirrhosis +* }\end{array}$ & $\begin{array}{c}\text { Road } \\
\text { accidents } \#\end{array}$ \\
\hline \multicolumn{8}{|l|}{ Urban (6) } \\
\hline Mean & 65.2 & 27.6 & 26.3 & 65.5 & 46.0 & 17.8 & 23.2 \\
\hline SD & 28.0 & 16.0 & 16.7 & 56.4 & 17.8 & 5.7 & 16.0 \\
\hline \multicolumn{8}{|l|}{ Upper Egypt(7) } \\
\hline Mean & 21.0 & 26.2 & 24.4 & 16.6 & 49.9 & 28.1 & 11.3 \\
\hline SD & 5.5 & 9.7 & 19.9 & 6.1 & 17.8 & 13.0 & 5.4 \\
\hline \multicolumn{8}{|l|}{ Lower Egypt(8) } \\
\hline Mean & 31.3 & 22.1 & 18.6 & 45.8 & 43.0 & 43.5 & 9.3 \\
\hline SD & 19.0 & 22.3 & 10.4 & 41.5 & 7.9 & 17.0 & 3.8 \\
\hline \multicolumn{8}{|l|}{ Frontiers(6) } \\
\hline Mean & 11.3 & 47.7 & 9.0 & 8.1 & 37.8 & 5.3 & 29.5 \\
\hline SD & 6.2 & 39.8 & 5.8 & 9.3 & 22.3 & 5.2 & 33.0 \\
\hline Total(27) & 31.7 & 30.1 & 19.7 & 34.2 & 44.3 & 25.3 & 17.4 \\
\hline $\begin{array}{l}\text { Mean } \\
\text { SD }\end{array}$ & 25.4 & 24.6 & 14.9 & 40.1 & 16.3 & 18.4 & 18.5 \\
\hline
\end{tabular}

\# Kruskal-Wallis Test

+ ANOVA Test

${ }^{*} P$-value $<0.05$

Table 3b. Correlation analysis between the deaths/100 000 population from the major noncommunicable diseases and percent achievement of the target number of general hospitals, ambulances and intensive care units, 2014

\begin{tabular}{|c|c|c|c|c|c|}
\hline Percent & Cancer deaths & $\begin{array}{l}\text { Hypertensive } \\
\text { diseases deaths }\end{array}$ & $\begin{array}{l}\text { Cerebro- } \\
\text { vascular } \\
\text { diseases }\end{array}$ & $\begin{array}{l}\text { Ischemic heart } \\
\text { diseases }\end{array}$ & Liver cirrhosis \\
\hline $\begin{array}{l}\text { Percent achievement of the target of } \\
\text { General Hospitals }\end{array}$ & $-0 \cdot 178$ & $-0 \cdot 226$ & $-0 \cdot 167$ & $-0 \cdot 491^{*}$ & $-0.598^{*}$ \\
\hline $\begin{array}{l}\text { Percent achievement of the target } \\
\text { Ambulances }\end{array}$ & $-0 \cdot 343$ & $-0 \cdot 376$ & $-0 \cdot 198$ & $-0.548^{*}$ & $-0.675^{*}$ \\
\hline $\begin{array}{l}\text { Percent achievement of the target } \\
\text { ICUs }\end{array}$ & $-0.415^{*}$ & $-0 \cdot 311$ & $-0 \cdot 177$ & $-0.605^{*}$ & $-0.475^{*}$ \\
\hline
\end{tabular}

Spearman's Rho Correlation Coefficient

${ }^{*}$ - value $<0.05$

Source: MoHP Health Statistics 
Table 4. Maternal mortality ratio (MMR) and child mortality by governorates, 2014

\begin{tabular}{|c|c|c|c|}
\hline Governorates & Neonatal mortality rate* & Under 5 mortality rate* & MMR by place of residence ** \\
\hline Cairo & 11.0 & 17.0 & 84.0 \\
\hline Alexandria & 19.0 & 27.0 & 50.0 \\
\hline Port-Said & 11.0 & 22.0 & 35.0 \\
\hline Suez & 16.0 & 21.0 & 32.0 \\
\hline Damietta & 8.0 & 15.0 & 42.0 \\
\hline Dakahlia & 13.0 & 22.0 & 57.0 \\
\hline Sharkia & 21.0 & 35.0 & 54.0 \\
\hline Kalyoubia & 19.0 & 39.0 & 59.0 \\
\hline Kafr-ElSheikh & 9.0 & 22.0 & 53.0 \\
\hline Gharbia & 17.0 & 29.0 & 60.0 \\
\hline Menoufia & 9.0 & 17.0 & 50.0 \\
\hline Behera & 11.0 & 19.0 & 56.0 \\
\hline Ismailia & 22.0 & 38.0 & 37.0 \\
\hline Giza & 10.0 & 25.0 & 53.0 \\
\hline Beni-Suef & 26.0 & 43.0 & 69.0 \\
\hline Fayoum & 14.0 & 25.0 & 59.0 \\
\hline Menia & 17.0 & 42.0 & 62.0 \\
\hline Asyout & 28.0 & 50.0 & 65.0 \\
\hline Suhag & 27.0 & 47.0 & 53.0 \\
\hline Qena & 16.0 & 38.0 & 58.0 \\
\hline Aswan & 26.0 & 35.0 & 51.0 \\
\hline Luxor & 25.0 & 48.0 & 24.0 \\
\hline Red Sea & 17.0 & 28.0 & 61.0 \\
\hline ElWadi ElGidid & 8.0 & 28.0 & 32.0 \\
\hline Matrouh & 9.0 & 21.0 & 71.0 \\
\hline${ }^{* * *}$ North Sinai & & & 34.0 \\
\hline${ }^{* * *}$ South Sinai & & & 0.0 \\
\hline Total & 14.0 & 27.0 & $52 \cdot 5$ \\
\hline
\end{tabular}

${ }^{*}$ Source: EDHS, 2014

** Source: MOHP statistics, 2014

${ }^{* * *}$ Not included in EDHS, 2014

Table 4a. Correlation analysis between MMR, Number of PHC/10 ooo population and percent achievement of the target of general hospitals and ambulances, 2014

\begin{tabular}{|c|c|c|c|}
\hline MMR & PHC/10000 population & $\begin{array}{l}\text { Percent achievement } \\
\text { of the target of general } \\
\text { hospitals }\end{array}$ & $\begin{array}{l}\text { Percent achievement of } \\
\text { the target of ambulances }\end{array}$ \\
\hline Spearman's Rho Correlation Coefficient & $-0 \cdot 44$ & -0.59 & -0.61 \\
\hline$P$-value & 0.022 & 0.001 & 0.001 \\
\hline
\end{tabular}

Spearman's Rho Correlation Coefficient

${ }^{*} \mathrm{P}$ - value $<0.05$

Source: MoHP Health Statistics 


\begin{tabular}{|c|c|c|c|c|c|c|}
\hline Prevalence of HBV and $\mathrm{HCV}^{*}$ & \multicolumn{4}{|c|}{ Hepatitis B virus (\%) } & \multicolumn{2}{|c|}{ Hepatitis C virus (\%) } \\
\hline Urban Governorates & \multicolumn{4}{|c|}{1.7} & \multicolumn{2}{|c|}{3.0} \\
\hline Lower Egypt & \multicolumn{4}{|c|}{0.7} & \multicolumn{2}{|c|}{5.6} \\
\hline Upper Egypt & \multicolumn{4}{|c|}{1.2} & \multicolumn{2}{|c|}{3.4} \\
\hline Frontier & \multicolumn{4}{|c|}{0.6} & \multicolumn{2}{|c|}{1.6} \\
\hline Total 1-59 Yrs & \multicolumn{4}{|c|}{1.0} & \multicolumn{2}{|c|}{4.4} \\
\hline Cancer** & \multicolumn{4}{|c|}{ Crude incidence rate / 100000} & & \\
\hline Lower Egypt ${ }^{* * *}$ & \multicolumn{4}{|c|}{133.5} & & \\
\hline Middle Egypt*** & \multicolumn{4}{|c|}{101.6} & & \\
\hline Upper Egypt ${ }^{* * *}$ & \multicolumn{4}{|c|}{105.5} & & \\
\hline Calculated rates of Egypt & \multicolumn{4}{|c|}{113.1} & & \\
\hline \multirow[t]{2}{*}{ Smoking, obesity and hypertension* } & \multicolumn{2}{|c|}{$\begin{array}{l}\text { Current smoking } \\
\text { prevalence (\%) }\end{array}$} & \multicolumn{2}{|c|}{ Obesity prevalence (\%) } & \multicolumn{2}{|c|}{$\begin{array}{c}\text { Prevalence of } \\
\text { hypertension (\%) }\end{array}$} \\
\hline & Female & Male & Female & Male & Female & Male \\
\hline Urban Governorates & 0.7 & 51.2 & 56.5 & 26.9 & 18.1 & 17.1 \\
\hline Lower Egypt & 0.1 & 44.3 & 55.4 & 30.5 & 18.0 & 16.7 \\
\hline Upper Egypt & 0.2 & 47.5 & 41.4 & 20.6 & 15.8 & 16.7 \\
\hline Frontier & 0.1 & 44.4 & 39.4 & 19.9 & 12.4 & 14.0 \\
\hline Total 15-59 yrs & \multicolumn{4}{|c|}{20.9} & 17.2 & 16.7 \\
\hline
\end{tabular}

*Source: EDHS, 2014

** Source: Cancer Registry Program, 2014

${ }^{* * *}$ Egypt was stratified into 3 geographical strata (regions) namely Lower Egypt (north of Cairo), Middle Egypt (south of Cairo) and Upper Egypt (further south, reaching the southern frontier of the country)

\section{Fertility}

A total fertility rate (TFR) of 3.5 child/ woman was reported in Egypt in 2014, and 20.8 years old was the mean age at first marriage for Egyptian females (4) (Table 2).

In almost all examined service coverage and availability indicators, the poorest performance was observed in Upper Egypt governorates (Table 1a, Table 2a).

\section{Discussion}

This study portrayed health services and outcomes in different geographical areas in Egypt, which is crucial to provide evidence for informed policy actions and for promoting health equity. There was a reduction in the number of health facilities/10 ooo population compared to 2001, which could be explained by the rapid population growth (9). The Egyptian population is growing at a rate of $2 \cdot 1 \%$ - higher than rates found in other Arab countries (2\%) (11). Furthermore, there were 15 beds for every 10000 population in 2014, which equals a bed for every 672 Egyptians compared to a bed for every 455 Egyptians in 2001 (1). This national average is higher than in some countries such as Pakistan and Iraq and lower than many middle-income countries (11). It is noteworthy that the bed capacity of the MoHP sector is coupled with bed occupancy rates $(50 \%)$, which places significant financial burden on the healthcare system and prevents the MoHP from allocating necessary resources to primary and preventive care (1).

There was in general a low achievement of national targets for service availability as well as wide geographical disparities. The least service availability and coverage was generally observed in the region of Upper Egypt. Furthermore, the number of ambulances, ICUs and NICUs were very high in governorates such as North and South Sinai and Red Sea, where the numbers exceeded the needs of these regions. However, careful assessment of coverage is crucial given the large surface area of such governorates coupled by the specific characteristics of the population served (12).

There was a high coverage of key $\mathrm{MCH}$ services examined in the current study attributed to the ongoing polit- 
ical commitment to improving $\mathrm{MCH}$. However, the last 6 years witnessed a decrease in the contraceptive prevalence rate which resulted in an increase in TFR and crude birth rate. In 2014, TFR increased to 3.5 live births, which is equivalent to the rate reported during 1990 (4). Inequitable coverage of $\mathrm{MCH}$ services was noted and was consistent with previous research $(1,4,12)$. In addition, early marriage was widespread in Egypt with approximately $6 \%$ of women in the age group 15-17 years currently or ever married (4). Increased fertility, increased population growth rate and persistent high level of early marriage highlight the utmost need to adopt and implement evidence-based strategies to reduce population growth and improve population characteristics.

There was a tangible increase in the MoHP workforce in 2014 (12). For example, the number of physicians increased from 6.5 in 2005 to 8.4 physicians / 10000 population in 2014 (12). Furthermore, there was nearly one physician / bed at MoHP services, which if combined with bed occupancy rate of $50 \%$, equals more than two physicians / occupied bed; this is much higher than many countries of similar economic status (1). This finding discounts claims regarding shortage in the number of physicians and underscores the problem of shortages in certain areas an indication of maldistribution of physicians rather than an actual shortage $(1,12)$.

Noncommunicable diseases, including cardiovascular diseases, diabetes, cancer, and chronic respiratory diseases, are currently the leading national cause of death in Egypt. Noncommunicable diseases are estimated to account for $82 \%$ of all deaths in Egypt (12). Cardiovascular disease mortality rates were the highest among NCDs, which is consistent with previous reports as well as the overall picture in all Arab countries $(13,14)$.

Egypt was successful in achieving Millennium DevelopmentGoal(MDG)4for reduction of childmortality and is on track to achieve its MDG 5a for reduction of maternal mortality (4). This decline is likely attributed to improved antenatal care and skilled birth attendance services as reported by EDHS 2014 (4). However, regional inequality in the provision of healthcare, the unequal distribution of trained health providers, the deficiency in the number of emergency obstetric care facilities, and the delay in referral for emergency obstetric services are still among the challenges threatening maternal health (15). This is supported by our finding that revealed MMR was significantly negatively correlated with both the number of PHC facilities/10 0oo, the percent achievement of the target number of general hospitals and ambulances.

In our study, many health outcomes were associated with health service availability indicators. For example, significant negative correlations were found between all service availability indicators and deaths due to ischemic heart disease and liver cirrhosis. Furthermore, there were evident geographical disparities in both health service availability and health outcome indicators. Putting all information together portrays a picture of inequality in health service availability which impacts health outcomes in the same inequitable pattern (15).
There is marked reduction in the incidence of major CDs in Egypt, particularly vaccine preventable diseases. However, bilharziasis incidence was the highest among all diseases under surveillance by MoHP. This is consistent with studies highlighting that Egypt is one of the countries with the highest number of cases of bilharziasis in the Middle East and North Africa region (16). Yet, it is important to highlight that the many years of efforts in Egypt have had a drastic impact on bilharziasis prevalence and endemicity. Studies in Egypt have demonstrated the effectiveness of the single dose administration of praziquantel - selective population chemotherapy that started in 1983. The MoHP reported that from 1982 to 1992 the prevalence of Schistosoma haematobium declined from around $15 \%$ to $1 \%$ in the Nile Delta and from 13\% to 3\% in Upper Egypt; the prevalence of Schistosoma mansoni declined from about $40 \%$ to $20 \%$ in the Nile Delta (17). In 2010, with an estimated countrywide prevalence of less than $0.3 \%$, another elimination effort was launched integrating sanitation, environmental interventions and health education into preventive chemotherapy campaigns (18). Currently, new strategies for combating bilharziasis based on sensitive surveillance tools need to be adopted to prevent resurgence and recrudescence.

The prevalence of hepatitis $\mathrm{C}$ viral $(\mathrm{HCV})$ infection showed a significant reduction among people aged 15-59 years, from $9.8 \%$ in 2008 to $7.0 \%$ in 2014 (4). There were many factors behind the reduction of HCV epidemic in Egypt including the introduction of Pegylated interferon (PEG-INF) (19) and later the introduction of Sofosbuvir to treat patients at a cost lower than $1 \%$ of its international price in 2014 (12).

The national age standardized incidence rate for all cancers in Egypt was 166.6/100 ooo for both sexes, which is intermediate compared to rates of higher and lowerincome countries. It is noteworthy that the distribution of liver cancer in the Egypt followed the distribution of HCV, which is more frequent in the Nile Delta with decreasing prevalence when moving south (19-21).

Tobacco use is increasingly prevalent in Egypt. Tobacco prevalence increased from $19.4 \%$ in 2009 to $20.9 \%$ in 2014 with the highest prevalence reported among males $(46 \cdot 4 \%)(4,22)$, and is a primary cause for the rising epidemic of NCDs in the country. Several effective tobacco control policies have been adopted and enacted. However, poor enforcement remains an unresolved challenge (12).

In Egypt, as in many Arab countries, obesity in adults has increased alarmingly (14), reaching 50\% in females compared to $26 \%$ in males (4). This gender difference in obesity might be reflected in the epidemiology of NCDs in Egypt with expected increasing prevalence of NCDs among females. Supporting this were the results of the EDHS 2014 that showed slightly higher prevalence of hypertension among females compared to the results of Stepwise Survey 2012, which reported higher prevalence among males $(4,23)$. 
The MoHP has made tangible efforts to reduce the burden of NCDs. A dedicated unit for NCDs was established in 2014, and an operational multi-sectoral national strategy that integrates major NCDs and their shared risk factors was developed. A national cancer committee to oversee cancer prevention and control was also established and developed evidence-based national guidelines for the treatment of the most prevailing cancers in Egypt (12). However, the surveillance system for NCDs and their risk factors is not yet institutionalized, is fragmented and relies mostly on sporadic national surveys. Furthermore, specific prevention programmes for NCD risk factors are rarely exist or implemented, since most government funds are allocated to treatment rather than prevention services (24). Therefore, government must prioritize the prevention and control of NCDs and their major risk factors with proper budget allocation in order to effectively prevent the rise in NCD morbidity and mortality.

\section{Conclusion}

This study sheds light on the current situation of health and health services in Egypt and the geographical distribution of key health services and outcomes. However, ecological fallacy, multiplicity of data sources, lack of assessment of health service quality, lack of data on the private sector, and absence of disaggregated data for some indicators due to either lack of data or limited access to such data, were among the key challenges of this study.

\section{Acknowledgements}

We acknowledge the welcome support and information sharing by the different sectors of MoHP. We particularly appreciate the efforts of Dr Amr Kandil, Head of the Preventive Sector; Dr Emad Ezzat, Head of Primary Health Care Sector; Dr Tarek Saad, Mrs Ibtesam Zakzouk and Dr Amany Gad, Central Department of Information; and Dr Yasser Omar, Dr Mohamed Gamal Eldin, Dr Mohamed El Teriaky, Dr Makram Atef, Dr Suzan Zanaty, Dr Mariam El Debeiky and Dr Sally Islam, Minister of Health Technical Office.

Funding: None.

Competing interests: None declared.

\section{La carte sanitaire égyptienne : une aide à la prise de décision fondée sur des données probantes}

\section{Résumé}

Contexte : Le système de santé égyptien se caractérise par la multiplicité des entités chargées de la réglementation, du financement et de la prestation de services. En outre, les résultats sanitaires en Égypte reflètent la transition épidémiologique; l'augmentation de la prévalence des maladies non transmissibles (MNT) et les niveaux élevés persistants de certaines maladies transmissibles font peser une énorme charge financière sur les ressources limitées du système de santé. Cette situation, conjuguée à l'inefficacité financière, aggrave les inégalités en matière de couverture des services de santé et de résultats sanitaires.

Objectifs : Notre objectif était de cartographier et de relier les services de santé aux principaux résultats sanitaires afin de favoriser l'utilisation efficace des ressources limitées et de réaliser la couverture sanitaire universelle (CSU).

Méthodes : Les données sur les services de santé et les résultats sanitaires ont été obtenues du ministère de la Santé et de la Population ainsi qu'à partir des principales enquêtes nationales pour l'année 2014. Les données sur la disponibilité et la couverture des services de santé ont été comparées dans différentes régions géographiques. Une analyse de corrélation a été utilisée pour tester la relation entre certains indicateurs de disponibilité des services et les taux de mortalité due aux principales MNT.

Résultats : Les principaux services de santé maternelle et infantile étaient largement couverts. Toutefois, dans l'ensemble, les objectifs nationaux en matière de disponibilité des services n'ont guère été atteints et les disparités géographiques étaient importantes. Les maladies cardio-vasculaires représentaient la principale cause de décès et l'incidence des principales maladies chroniques en Égypte, en particulier des maladies à prévention vaccinale, a nettement diminué.

Conclusions : Les larges disparités géographiques et l'augmentation de la charge des maladies non transmissibles et de leurs facteurs de risque sont identifiées comme les principaux défis auxquels le système de santé égyptien est confronté. L'établissement des priorités à la lumière de ces résultats est crucial pour appuyer les efforts nationaux visant à atteindre l'équité en santé et réaliser la CSU. 


\section{خريطة الصحة المصرية: دليل اتخاذ القرارات المسند بالبراهين \\ غادة رضوان، عادل العدوي}

الخلاصة

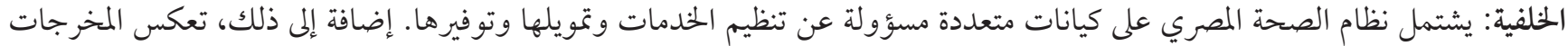

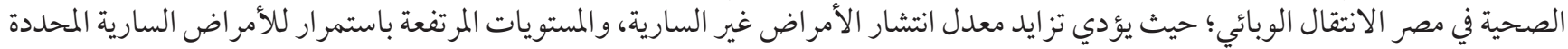

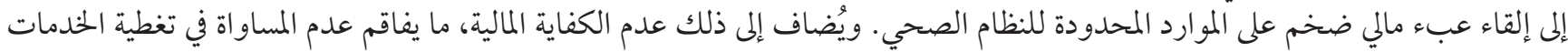
الصحية والمخرجات الصحية.

الأهداف: نهدف إلى وضع خريطة للخدمات الصحية وربطها بالمخرجات الصحية الرئيسية لدعم الاستخدام الفَّال للموارد الضئيلة وبلوغ التغطية الصحية الشاملة.

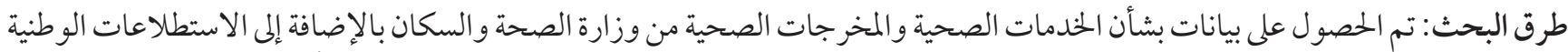

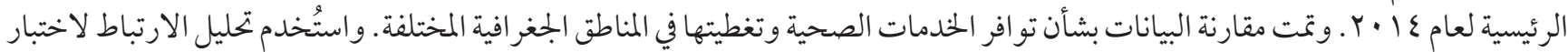

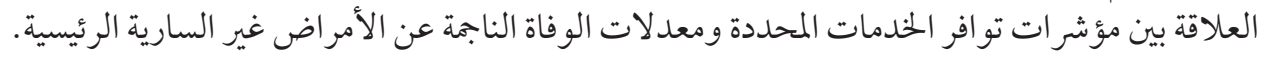

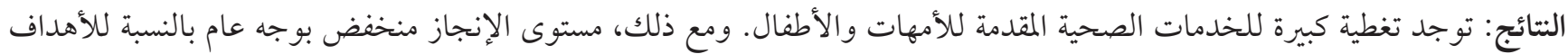

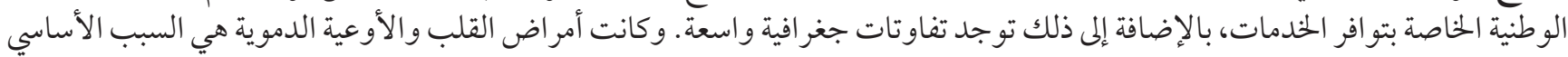

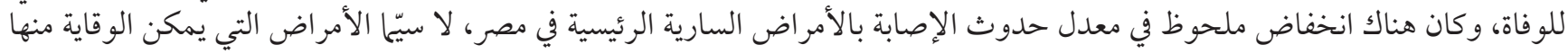
باللقاحات.

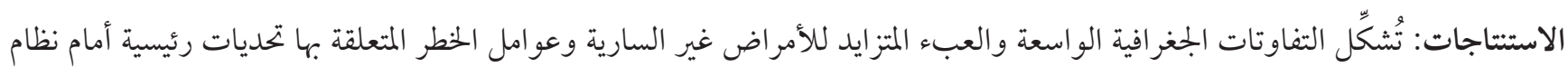

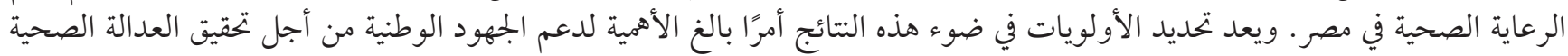
والتغطية الصحية الشاملة.

\section{References}

1. World Health Organization. Health System Profile in Egypt. Geneva: World Health Organization; 2006. (http://apps.who.int/ medicinedocs/documents/s17293e/s17293e.pdf).

2. World Health Organization. White Paper: Framing National Health Policy. Geneva: World health Organization; 2014.

3. Handler A, Issel M, Turnock B. A Conceptual Framework to Measure Performance of the Public Health System. Am J Public Health. 2001;91(8):1235-1239. PMID: 11499110

4. Ministry of Health and Population [Egypt], El-Zanaty and Associates [Egypt], and ICF International. Egypt Demographic and Health Survey 2014. Cairo and Rockville: Ministry of Health and Population and ICF International, 2015.

5. National Cancer Registry Program of Egypt (NCRPE) 2017. (http://cancerregistry.gov.eg/).

6. World Health Organization. World Health Statistics 2015: Indicator compendium. Geneva: World Health Organization; 2015. (http://www.who.int/gho/publications/world_health_statistics/WHS2015_IndicatorCompendium.pdf).

7. World Health Organization. Health service delivery. Geneva: World Health Organization; 2010 (http://www.who.int/healthinfo/ systems/WHO_MBHSS_2010_section1_web.pdf?ua=1).

8. World Health Organization. WHO Statistical Information System (WHOSIS). Geneva: World Health Organization; 2017 (http:// www.who.int/whosis/en/).

9. Central Agency for Public Mobilization and Statistics (CAPMAS). Cairo: CAMPAS; 2017 (http://www.capmas.gov.eg/Pages/StaticPages.aspx?page_id=5034)

10. World Health Organization. The global health expenditure database. Geneva: World Health Organization; 2017 (http://apps.who. int/nha/database/ViewData/Indicators/en).

11. World Development Indicators: Washington, DC: World Bank, 2017 (http://databank.worldbank.org/data/reports.aspx?source $=2 \&$ series $=$ SH.MED.BEDS.ZS\&country $=$ ).

12. World Health Organization Regional Office for Eastern Mediterranean (WHO/EMRO). Egypt health profile, WHO/EMRO; 2015. (http://applications.emro.who.int/dsaf/EMROPUB_2016_EN_19264.pdf?ua=1\&ua=1).

13. Global burden of diseases, injuries, and risk factors study 2010. Seattle, WA: Institute for Health Metrics and Evaluation; 2010.

14. Mokdad AH, Jaber S, Aziz MI, AlBuhairan F, AlGhaithi A, AlHamad NM, et al. The state of health in the Arab world, 1990-2010: an analysis of the burden of diseases, injuries, and risk factors. Lancet. 2014;383(9914):309-20. https://doi.org/10.1016/So1406736(13)62189-3 PMID: 24452042 
15. Lindtjorn B, Mitiku D, Zidda Z, Yaya Y. Reducing Maternal Deaths in Ethiopia: Results of an Intervention Programme in Southwest Ethiopia. PLoS One. 2017;12(1):e0169304. https://doi.org/10.1371/journal.pone.0169304 PMID: 28046036

16. Hotez PJ, Savioli L, Fenwick A. Neglected tropical diseases of the Middle East and North Africa: review of their prevalence, distribution, and opportunities for control. PLoS Negl Trop Dis. 2012;6(2):e1475. https://doi.org/10.1371/journal.pntd.0001475

17. International strategies for tropical disease treatments. Experiences with praziquantel. Geneva: World Health Organization; 1998. (http://apps.who.int/medicinedocs/en/d/Jwhozip48e/8.2.html\#Jwhozip48e.8.2).

18. World Health Organization. 2013. Schistosomiasis: progress report 2001-2011, strategic plan 2012-2020. Geneva: World Health Organization; 2013 (apps.who.int/medicinedocs/en/d/Jwhozip48e/8.2.html)

19. Elgharably A, Gomaa AI, Crossey MM, Norsworthy PJ, Waked I, Taylor-Robinson SD. Hepatitis C in Egypt - past, present, and future. Int J Gen Med. 2017;10:1-6. https://doi.org/10.2147/IJGM.S119301 PMID: 28053553

20. Ezzat S, Abdel-Hamid M, Eissa SA, Mokhtar N, Labib NA, El-Ghorory L, et al. Associations of pesticides, HCV, HBV, and hepatocellular carcinoma in Egypt. Int J Hyg Environ Health. 2005;208(5):329-39. PMID: 16217918

21. Ibrahim AS, Khaled HM, Mikhail NN, Baraka H, Kamel H. Cancer incidence in Egypt: results of the national population-based cancer registry program. J Cancer Epidemiol. 2014;2014:437971. https://doi.org/10.1155/2014/437971 PMID: 25328522

22. World Health Organization Regional Office for the Eastern Mediterranean (WHO/EMRO). Global adult tobacco survey: Egypt country report 2009. Cairo: WHO/EMRO; 2009 (http://www.who.int/tobacco/surveillance/gats_rep_egypt.pdf).

23. World Health Organization. Egypt STEPS Survey 2011-12. (http://www.who.int/chp/steps/2011-2012_Egypt_FactSheet.pdf).

24. World Health Organization Regional Office for the Eastern Mediterranean (WHO/EMRO). Assessing national capacity for the prevention and control of noncommunicable diseases: report of the 2015 country capacity survey in the Eastern Mediterranean Region. Cairo: WHO/EMRO; 2015 